\title{
LA EFICIENCIA TECNICA EN LAS TERMINALES DE CONTENEDORES A PARTIR DE LA LEY DE BENFORD: Aplicación a los puertos españoles
}

\author{
Miguel Angel Pesquera González
}

University of Cantabria, Department of transportation; Av. de los Castros, S/N. 39005

Santander (Cantabria). Spain. E-mail: miguel.pesquera@unican.es

\section{Pablo Coto Millán}

University of Cantabria, Department of applied economics, Av. de los Castros, S/N. 39005

Santander (Cantabria).Spain. E-mail: pedro.coto@unican.es

\section{Benito Díaz Medina}

University of Cantabria, Department of applied economics, Av. de los Castros, S/N. 39005 Santander (Cantabria).Spain. E-mail: benitodm90@gmail.com

\section{Juan Castanedo Galán}

University of Cantabria, Department of transportation; Av. de los Castros, S/N. 39005

Santander (Cantabria). Spain. E-mail: juan.castanedo@unican.es

\section{RESUMEN}

Las terminales de contenedores en los puertos son los nodos del sistema de transportes derivado de un sistema abierto y complejo como es el del flujo de las mercancías en el intercambio de bienes.

La eficiencia técnica se da en economía, utilizando todos sus recursos de manera eficiente, produciendo el máximo de producción con el mínimo de recursos.

En la presente ponencia se demuestra que la función de distribución de equilibrio de los movimiento de contenedores en los puertos se ajusta bien a la denominada ley de Benford, del primer digito, o de distribución desigual de dígitos en datos numéricos, ley de potencia que se obtiene como resultado de maximizar la entropía de Shannon, lo que significa obtener la mayor eficiencia técnica.

Del ajuste de la ley de Benford con los resultados obtenidos para los puertos españoles en los tráficos totales y de contenedores, nos abre una nueva línea de investigación para comprobar la eficiencia de los puertos y poder plantear mejoras para su funcionamiento.

\section{1.- INTRODUCCION: Ley de Benford.}

La ley del primer dígito, conocida como ley de Benford, es un fenómeno matemático sorprendente, como señala Parrondo (2002), que revela una cierta universalidad en los aspectos cuantitativos del mundo, y una propiedad común a series de datos que provienen de fuentes muy diversas.

En el siglo XIX los científicos e ingenieros realizaban cálculos complejos gracias a los logaritmos. Para multiplicar dos números bastaba averiguar sus logaritmos, sumarlos y 
encontrar el antilogaritmo de esta suma. Los científicos consultaban constantemente sus tablas.

No fue Benford, sino un matemático y astrónomo llamado Newcomb el descubridor de la ley, al darse cuenta de que las primeras páginas de las tablas de logaritmos de la biblioteca de su facultad estaban mucho más sucias y usadas que las últimas. Fue a la biblioteca de una facultad diferente a la suya, y el resultado fue el mismo los números que comienzan por el digito uno eran más consultados que los que comienzan por nueve.

El propio Newcomb encontró, una fórmula para la probabilidad de que un número en una serie de datos comience por el dígito $\mathrm{d}$.

La fórmula es:

$$
\mathrm{Pd}=\log _{10}(1+1 / \mathrm{d})
$$

Utilizando esta fórmula se encuentra que, para $\mathrm{d}=1$ la probabilidad es, del 30,1\%, para $\mathrm{d}=2$ del $17,61 \%$, para $\mathrm{d}=3 \mathrm{del} 12,46 \%$, para $\mathrm{d}=4$ del $9,69 \%$, para $\mathrm{d}=5 \mathrm{del} 7,92 \%$, para $\mathrm{d}=6$ del $6,69 \%$, para $\mathrm{d}=7 \mathrm{del} 5,80 \%$, para $\mathrm{d}=8 \mathrm{del} 5,12 \%$ y para $\mathrm{d}=9 \mathrm{del} 4,58 \%$.

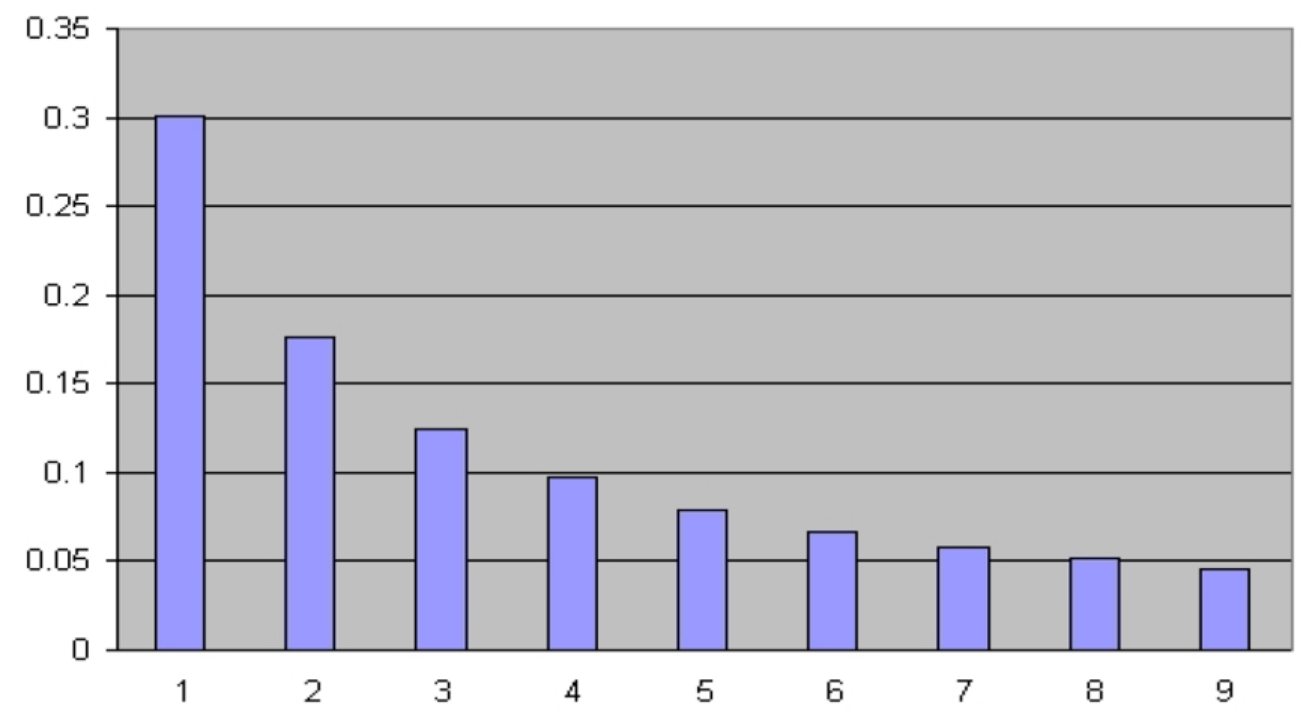

Figura 1. Ley de Benford o probabilidad decreciente de los primeros dígitos (1 al 9)

Hoy se conoce con el nombre de ley Benford por su célebre artículo "La ley de los Números anómalos" de 1932, con sus múltiples demostraciones prácticas y el impulso y desarrollo que la dio.

El mundo está lleno de sucesos que crecen exponencialmente, desde las células a las redes sociales. Cuando el crecimiento es natural, lo que también suele coincidir cuando nos encontramos con sistemas complejos y abiertos, como acontece con la logística y el transporte como resultado de la demanda derivada de distintos actos sociales, la mayoría de los datos que se pueden extraer verifican la distribución de Benford. 
Pinkham (2015) ha demostrado que la distribución logarítmica de Benford es invariante en escala, lo que significa poder utilizar todo tipo de datos para ajustarse a la Ley.

Como señala Poundstone (2015), en su libro "Como predecir lo impredecible" en el contexto de los sistemas complejos, caso que nos ocupa en la presente ponencia en la aplicación de las cadenas logísticas y en particular en los movimientos de tráfico portuario: "Cuando la gente toma decisiones arbitrarias, al azar, o estratégicas, caen en patrones inconscientes que se pueden predecir" lo que viene a decir que podemos anticiparnos a predecir disfunciones/ desajustes con la ley de Benford, identificando posibles ineficiencias que nos oriente a su confirmación o rechazo con una más detallada investigación .

\section{2.- La Complejidad y las cadenas logísticas.}

La complejidad, como tal, no es fácil de definir. Desde una perspectiva de sistemas, la complejidad puede entenderse como una propiedad específica, definida como el resultado de la cantidad de elementos del sistema, sus relaciones y la dinámica entre sus elementos y sus relaciones. Cuanto más posibles estados se puedan encontrar en un sistema, mayor es la variedad del mismo. La variedad se puede usar entonces como una medida de la complejidad. En general, la complejidad se caracteriza por dos aspectos: la estructura y el comportamiento del sistema.

En general, la complejidad de una cadena logística puede ser descrita en términos de varias aspectos interconectados del sistema que la compone, incluyendo, ver Steve (2004):

- número de elementos o subsistemas;

- grado de orden dentro de la estructura de elementos o subsistemas;

- grado de interacción o la conectividad entre los elementos, subsistemas y el medio ambiente;

- nivel de variedad, en cuanto a los diferentes tipos de elementos, subsistemas, y las interacciones; y

- grado de predictibilidad y la incertidumbre dentro del sistema.

Hay referencias recientes, Hales (2009), que tratan sobre la aplicabilidad de la ley de Benford para obtener mejoras en los procesos de decisión de las cadenas logísticas. Basadas en identificar los funcionamientos de ineficiencia y usos fraudulentos en alguno de los agentes económicos de la cadena. Identificando estas anomalías pueden tomarse decisiones que permitan mejoras de eficiencia en el conjunto de las cadenas logísticas.

\section{3.- Los puertos como sistemas complejos.}

Las operaciones que se realizan a través de las terminales portuarias de contenedores se consideran entre las tareas más complejas dentro del sector del transporte, como consecuencia de las siguientes características: 
- Interacciones complejas en los aspectos físicos y de información, dado el número de los diferentes agentes que están involucrados en las importaciones y exportaciones de contenedores, como en las compañías marítimas, ferroviarias, agentes de aduana, compañías exportaciones, transportistas, etc.

- Interacciones complejas en las operaciones y los procesos que tienen lugar en el espacio portuario.

En la siguiente figura se ve claramente la complejidad desarrollada en el puerto con las operaciones desde el transporte marítimo al terrestre y las maniobras o movimientos realizados por los medios de transporte.

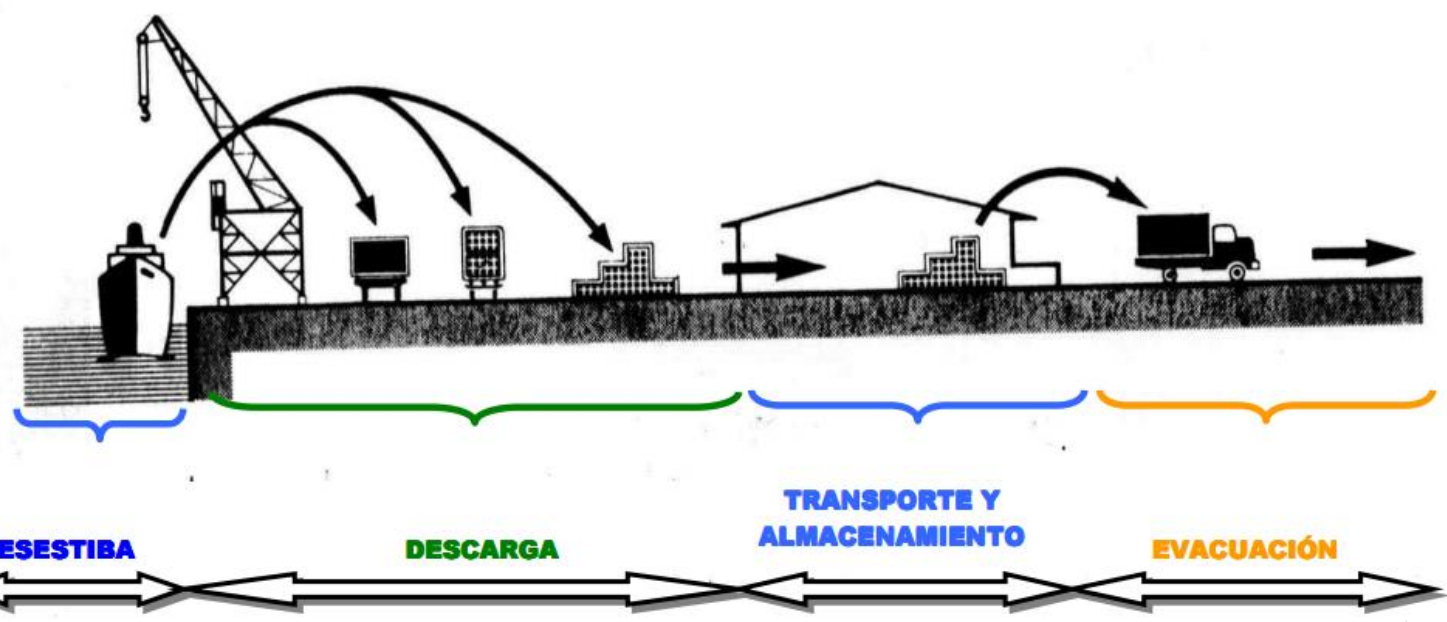

Figura 2.-Actividades que componen las Operaciones Portuarias y de la manipulación de mercancías en su paso por el puerto.

En la realización de las operaciones portuarias pueden distinguirse distintos tipos de acuerdo con el punto donde se desarrolla la operación, véase puertos del estado (2003):

- A bordo del barco: Operación en bodegas y cubiertas.

- De barco a barco: Operación que se lleva a cabo entre dos barcos a flote, empleado para ello una maquinaria y utillaje que optimiza el proceso y evita la pérdida de mercancía.

- Entre barco y tierra: Operación de transferencia de la mercancía entre ambos puntos (también se incluye la operación directa sobre el vehículo).

- En tierra: Operaciones de transporte y almacenamiento entre el punto de descarga del barco, así como las operaciones que se realizan con la mercancía antes o después del transporte marítimo.

- Entre tierra y transporte terrestres: Operaciones de carga y descarga del vehículo terrestre. 
La presente ponencia constituye la primera aplicación de la Ley de Benford a los sistemas de transporte y en concreto al sistema portuario. Lo que supone una aportación importante en el conocimiento y análisis de la eficiencia de los puertos desde otra perspectiva, diferente y sencilla de llevar a cabo, como a continuación veremos, y que nos abre un nuevo campo de investigación.

\section{4.- La Ley de Benford, como maximización de la entropía (Shannon): análisis de la eficiencia en tráficos portuarios totales y de contenedores en los Puertos españoles.}

La Teoría de la Información proporciona un medio de cuantificar la complejidad. La complejidad de un sistema aumenta con el aumento de los niveles de desorden y de incertidumbre. Por lo tanto, un sistema de complejidad superior requiere una mayor cantidad de información para describir su estado. Desde una perspectiva de teoría de la información, la entropía se define como la cantidad de información necesaria para describir el estado del sistema, Shannon (1948). Por lo tanto, un aumento en la complejidad de un sistema, mediante un mayor desorden e incertidumbre, aumenta su entropía, que puede ser medido como un aumento en la cantidad esperada de información requerida para describir el estado del sistema.

La entropía es una medida del desorden, Todos los estados físicos tienden al estado más probable y ese siempre es el que tiende a aumentar el desorden. La entropía crece en todos los proceso naturales, recuérdese la segunda ley de la termodinámica.

El equilibrio, o punto de mayor eficiencia se encuentra al aumentar el número de opciones disponibles lo que nos lleva a la maximización de la entropía, como resultado.

Kafri (2013) demuestra que la distribución de dígitos decimales que maximiza la entropía de Shannon (número de microestados) tiene como resultado la ley de Benford, y que tiene su correspondencia con actividades físicas y tangibles, más allá de las operaciones matemáticas. Esta importante realidad se demuestra en la presente ponencia con el ajuste de los tráficos portuarios y de contenedores en España, lo que significa obtener su punto de equilibrio o de eficiencia técnica.

Varian (1972) planteó la viabilidad de la Ley de Benford para evaluar la fiabilidad de previsiones. En los casos comprobados en donde las frecuencias de primer dígito se ajustaban a la Ley de Benford, las previsiones también siguieron la Ley de Benford, con lo que Varian (1972) concluyó que revisar los pronósticos con la Ley de Benford era una prueba potencial de la razonabilidad de las previsiones.

\section{Resultados de la Ley de Benford en su aplicación a los puertos españoles}

Disponemos de una serie histórica de tráficos portuarios totales, Puertos del Estado (2016), para los puertos de interés general españoles, desde el año de 1962 hasta 2015, lo que 
representa un panel de 1539 datos, suficiente para obtener su distribución del primer dígito, y ver su ajuste en relación a la ley de Benford, cuyo resultado se recoge en la figura 3, con un buen resultado de comportamiento.

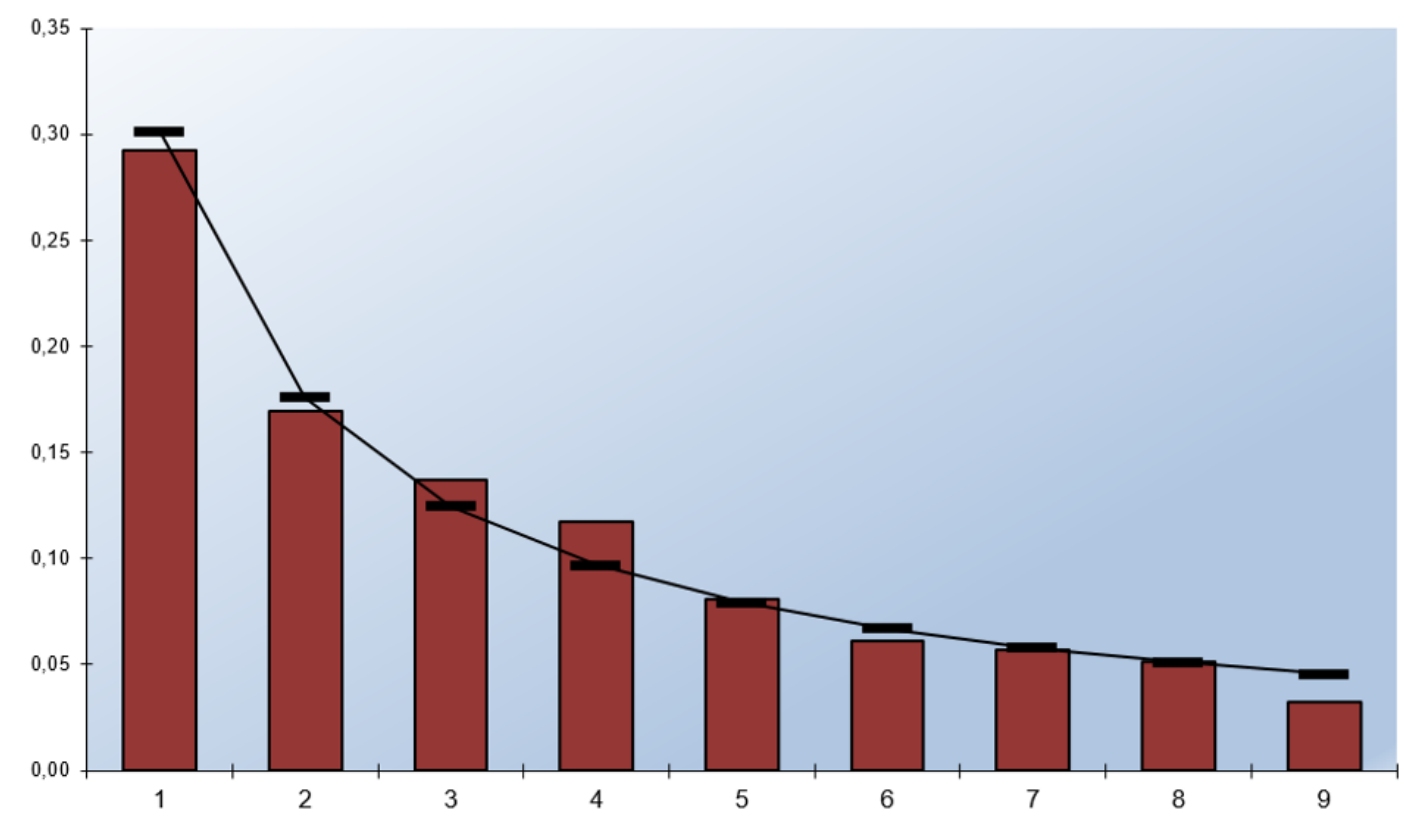

Figura 3.- Tráficos totales de los puertos españoles y su ajuste a la ley de Benford para el periodo comprendido entre 1962-2015

En relación a las terminales de contenedores se dispone de una serie histórica de tráficos de contenedores, Puertos del Estado (2016), en los puertos de interés general españoles, expresados en teus, desde el año de 1973 hasta 2015, recordemos que este tipo de tráfico es de más reciente implantación, con un panel de 1041 datos, suficiente para obtener su distribución del primer dígito, y ver su ajuste en relación a la ley de Benford, cuyo resultado se recoge en la figura 4 , con un ajuste algo inferior al obtenido con los tráficos totales.

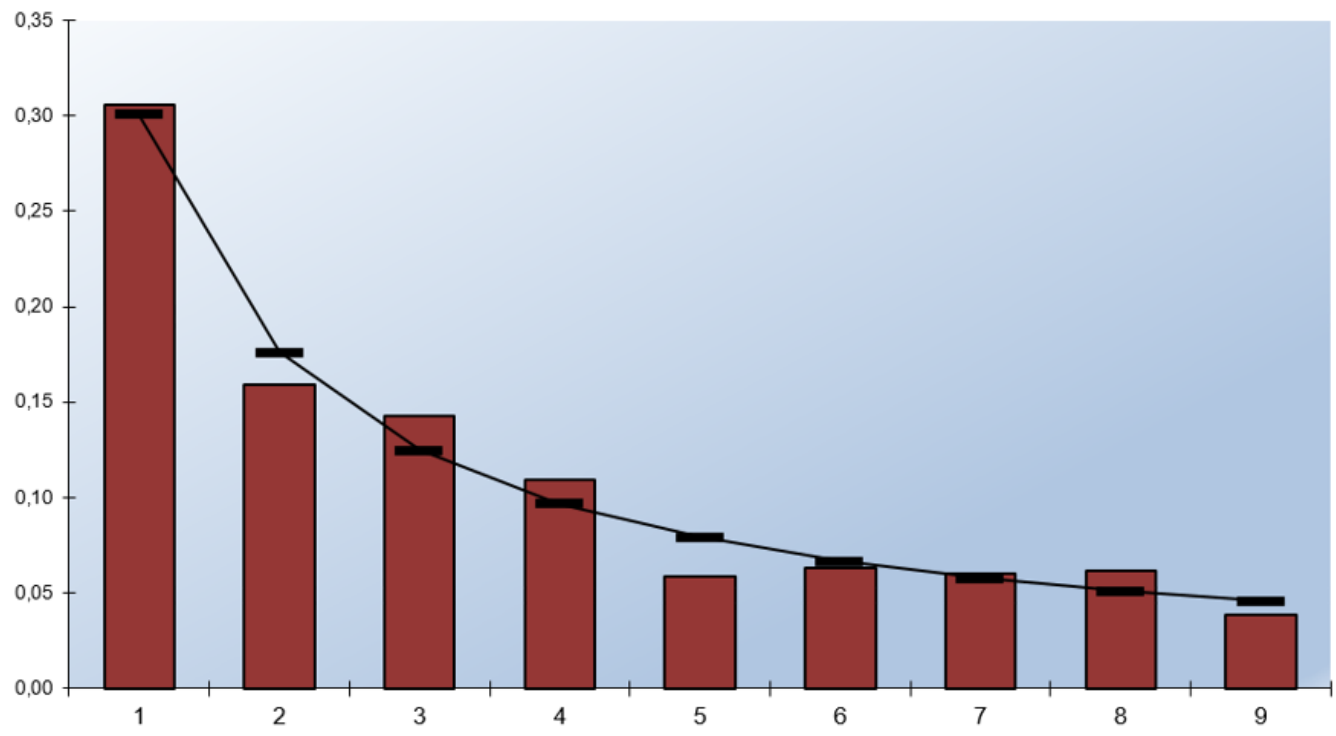




\section{Figura 4.- Tráficos de contenedores de los puertos españoles y su ajuste a la ley de Benford para el periodo comprendido entre 1973-2015}

\section{5.- CONCLUSIONES}

De lo estudiado e investigado, recogido en los apartados anteriores de la ponencia se resaltan las siguientes conclusiones;

A. Si bien, el sistema portuario en general y el español en particular tienen a crecer, gracias al sistema de relaciones comerciales internacionales, realizar predicciones con suficiente precisión y antelación, no es posible debido a su característica de sistema complejo. Sin embargo, podemos anticiparnos, y predecir comportamientos gracias al cumplimiento de la ley de Benford o del primer dígito.

B. La ley de Benford que cumple con la maximización de la entropía, recoge en su distribución los puntos de equilibrio o de mayor eficiencia técnica, hecho que se cumple y ajusta en su aplicación a los movimientos en los tráficos portuarios totales (periodo 1962-2015) y de contenedores (periodo 1973-2015) en los puertos españoles.

C. Los resultados del ajuste realizado a la ley del primer digito, para los movimientos portuarios en los puertos españoles, demuestra mayor eficiencia en los tráficos portuarios totales frente a los realizados en las terminales de contenedores, hecho que habría estudiar, como posibles cambios y singularidades en algún puerto, y con estudios del segundo digito de la ley de Benford.

D. La logística y los sistemas de transporte, son ámbitos adecuados, dado su complejidad, para abrir un nuevo campo de investigación en relación a la aplicación y ajustes a la ley de Benford, abriendo perspectivas metodológicas nuevas y sorprendentes.

E. En las previsiones realizadas en los planes de empresa de las autoridades portuarias, cuyas metodologías están basadas en datos y series históricas, así como en el conocimiento de nuevas actuaciones generadoras de tráficos, por el sistema portuario globalmente y por las propias autoridades portuarias, se pueden hacer previsiones con la ley de Benford siguiendo los planteamientos de Varian (1972).

F. Otra nueva línea de investigación que se puede a plantear se correspondería con investigar el comportamiento individual portuario para ordenar y anticipar los posibles fallos o ineficiencias técnicas. 


\section{REFERENCIAS}

Hales, D.; Chakravorty S. and Sridhara, V. (2009). Testing Benford's Law for improving supply chain decision-making: A field experiment. Int. J. Production Economics, 122, 606618.

Kafri , O. and Kafri, H. (2013). Entropy. God's Dice Game. Edited by Emanuel Lotem, USA.

Puertos del Estado, (2003). Ministerio de Fomento. Operaciones y Servicios Portuarios (nivel 1). http://www.apmarin.com/download/686_opsp1.pdf.

Puertos del Estado, (2016). Ministerio de Fomento. Estadísticas, http://www.puertos.es/eses/estadisticas/Paginas/estadistica_Historicas.aspx.

Parrondo, R. y Juan M. (2002). La misteriosa ley del primer dígito. Investigación y Ciencia, diciembre de 2002.

Pinkham, R. (1961). On the Distribution of First Significant Digits. The Annals of Mathematical Statistics, Vol. 32, No. 4.

Poundstone, W. (2014). How to Predict the Unpredictable. Oneworld Publications, London,

Shannon, C. E. (1948). A Mathematical Theory of Communication. Bell. System Technical Journal, 27: 379-423.

Steve, N. and Westbrook, R. (2004). Understanding Supply Chains. Oxford University Press. New York.

Varian, H. (1972). Benford's Law. The American Statistician, 23 (June), 65-66. 